\title{
SKA antenna systems; outlook for non-astronomy applications
}

\author{
A. van Ardenne ${ }^{1,2}$, M. J. Bentum ${ }^{1,3}$, A.J. Boonstra ${ }^{1}$ \\ ${ }^{1}$ ASTRON, P.O Box 2, 7990 AA Dwingeloo, The Netherlands, ardenne@astron.nl, boonstra@astron.nl \\ ${ }^{2}$ Chalmers Technical University, Gothenbörg, Sweden \\ ${ }^{3}$ Faculty Electr.Eng, Math. \& Comp.Sc., Twente University, P.O Box 217, 7500 AE Enschede, The Netherlands, \\ m.j.bentum@utwente.nl
}

\begin{abstract}
The globally endorsed Square Kilometre Array project primarily aims to advance high sensitivity radio astronomy using a distributed collection of radio telescope stations spiraling outward from the core along three to five arms out to $3000 \mathrm{~km}$. This planned highly sensitive instrument covering a frequency range from $70 \mathrm{MHz}$ up to $10 \mathrm{GHz}$ will be used as wideband, high resolution, wide observing field interferometer of which the first phase will be realized this decade. With these SKA telescope capabilities and with the underlying technologies, there are many space related applications outside the immediate radio astronomy domain especially in the ground segment area. Examples are tracking space debris, precision orbit determination, simultaneous deep space tracking of multiple spacecrafts, GNSS and for other ground segment applications such as search and rescue tracking.

After a brief introduction to the SKA, this paper will explore these potential application areas using the SKA based on its underlying approaches in the antenna and receiving subsystems.
\end{abstract}

\section{INTRODUCTION}

The Square Kilometre Array, SKA is the next generation low frequency radio telescope with preliminary specifications described by Dewdney et al. [1]. Its large collecting area is realized by a distributed and networked collection of radio telescope stations spiraling outward from the core along three to five arms out to $3000 \mathrm{~km}$. Each station is realized by multielement, so called aperture arrays stations or one reflector dish operating at higher frequencies. The work performed in the European funded FP6 programme, SKA Design Studies, SKADS, [2,3,4] showed that an implementation of the SKA using phased aperture arrays, AAs, operating from $70 \mathrm{MHz}$ up to $1.4 \mathrm{GHz}$ with a dish based array covering $\sim 1.2 \mathrm{GHz}$ to $10 \mathrm{GHz}$ represents the most capable design for the projected SKA science case [5]. As the final system is still being discussed, other implementation scenarios include the use of phased array feeds on dishes as field of view expansion technique with continuous beams allowing simultaneous wide field high efficiency observations [e.g. 6]. At the same time wideband feed studies for radio astronomical usage [e.g. 7] are ongoing possibly combined with focal plane array feeds.

The dishes presently being studied focus on smaller i.e. less than $15 \mathrm{~m}$ diameter, mass produced dishes making extensive use of composites materials in the reflector. As SKA aims to emulate a large collecting area, over 2000 dishes are to be produced at "low cost" and enabling use in relatively harsh, desert like conditions. This is because the SKA siting candidates in South Africa with its African partner countries and Western Australia with New Zealand are suitably located for example with respect to atmospheric and low radio interference level. The cores are extending from the Karoo in S.A. and the Murchison Shire in Western Australia with the spiral arms covering long baselines to enable high resolution observing with the SKA.

SKA activities are in all areas including Aperture Arrays (AA's), Focal plane arrays (FPA's) and feed systems. At system level knowledge is to be gained particularly from demonstrator projects. For dishes these are progressing in South Africa [8] as well as in Western Australia [9]. These are using offset and symmetric dishes respectively, the last making use of rotating subreflector to "freeze" the observing beam on the sky. At lower frequencies the large programs for Aperture Arrays are LOFAR [10] at low frequencies i.e. $30-240 \mathrm{MHz}$ and EMBRACE [11] from 500-1500MHz. L-band interferometric phased Focal Plane Arrays or Phased Arrays Feeds (PAF's) have been demonstrated by the APERTIF system at the Westerbork Synthesis Radio Telescope [6].

Timeline wise, the phased deployment of the SKA is planned to start with a $\sim 10 \%$ instrument in 2016 . It includes a low frequency sparse AA covering $70 \mathrm{MHz}-$ $\sim 450 \mathrm{MHz}$ besides several hundred smaller dishes. The development of the technically less mature dense AA system will continue in parallel preparing for deployment in SKA Phase 2 commencing in 2019. This schedule enables SKA Phase 1 to benefit from the experience gained with current low frequency arrays notably LOFAR and MWA [12] systems, followed by the more challenging dense AA in Phase 2. These array 
developments are core to the Aperture Array Verification Program [4, 13] advancing AA's for the SKA.

Table 1 below summarizes a range of realization scenarios of the SKA as studied today.

\begin{tabular}{|c|c|c|c|c|}
\hline Freq. Range & Collector & Sensitivity & Number / size & Distribution \\
\hline $\begin{array}{l}70 \mathrm{MHz} \\
\text { to } 450 \mathrm{MHz}\end{array}$ & $\begin{array}{l}\text { AA-low } \\
\text { Sparse AA }\end{array}$ & $\begin{array}{l}4,000 \mathrm{~m}^{2} / \mathrm{K} \text { at } \\
100 \mathrm{MHz}\end{array}$ & $\begin{array}{l}250 \text { array stations, } \\
\text { Diameter } 180 \mathrm{~m}\end{array}$ & \multirow{2}{*}{$\begin{array}{c}66 \% \text { within } 5 \mathrm{~km} \text { dia., } \\
34 \% \text { along } 5 \text { spiral } \\
\text { arms out to } \\
180 \mathrm{~km} \text { radius }\end{array}$} \\
\hline $\begin{array}{l}400 \mathrm{MHz} \\
\text { to } 1.45 \mathrm{GHz}\end{array}$ & $\begin{array}{l}\text { AA-mid } \\
\text { Dense AA }\end{array}$ & $\begin{array}{l}10,000 \mathrm{~m}^{2} / \mathrm{K} \text { at } \\
800 \mathrm{MHz}\end{array}$ & $\begin{array}{l}250 \text { array stations, } \\
\text { Diameter } 56 \mathrm{~m}\end{array}$ & \\
\hline $\begin{array}{l}300 / 1000 \mathrm{MHz} \\
\text { to } 10 \mathrm{GHz}\end{array}$ & $\begin{array}{l}\text { Dishes with } \\
\text { single pixel feed } \\
+ \text { PAF }\end{array}$ & $\begin{array}{l}10,000 \mathrm{~m}^{2} / \mathrm{K} \text { at } \\
1.4 \mathrm{GHz}\end{array}$ & $\begin{array}{l}2000-3000 \text { dishes } \\
\text { Diameter } 15 \mathrm{~m}\end{array}$ & $\begin{array}{l}50 \% \text { within } 5 \mathrm{~km} \mathrm{dia}, \\
30 \% 5 \mathrm{~km}-180 \mathrm{~km} \\
20 \% 180 \mathrm{~km}-3,000 \mathrm{~km} .\end{array}$ \\
\hline
\end{tabular}

Table 1: Summary of realization scenarios of the full SKA as studied today including Aperture Arrays ("AA") and Phased Array feeds ("PAF"). Sensitivity is expressed as the ratio of effective collecting area over system noise.

\section{ASTRONOMICAL POTENTIAL}

By design, the nature of the SKA is primarily intended to enable to resolve key physical question through astronomical discoveries. These are broadly related to (i) the origins of the universe through study of the neutral hydrogen in Doppler shift and astro-biology, (ii) the fundamental forces involving e.g. the study of pulsars, cosmic magnetic fields and gravitation and (iii) transients and exploration of the unknown [14].

As suggested in the Introduction huge steps are required to facilitate opening up SKA's discovery space requiring new functional capabilities to operate simultaneously. These include (i) wide (simultaneous) observing fields of up to tens of squared degrees i.e. 10100 times larger than the full moon, made possible by (simultaneous) multi-beaming (ii) wide instantaneous frequency bands of up to several $\mathrm{GHz}$ with (iii) high spatial resolution determined by the longest baseline (up to $3000 \mathrm{~km})$ at the highest frequency $(10 \mathrm{GHz})$ i.e. of order milli-arc second (iv) high dynamic range imaging techniques at (v) high sensitivity of 10-100 times larger than presently possible with radio telescopes.

The above is enabled by intense high-throughput signal processing [e.g. 15], broad-band networking and high performance computing capabilities and by new calibration and imaging techniques not specifically covered in this paper. Functionally these require low power beam hierarchical beam forming, data-streaming calibration and correlation and imaging techniques now being studied. Understandably the experiences gained from LOFAR [10] are relevant.

SKA's set of functional (observing) capabilities and the supporting technologies offer potential for nonastronomical applications in the space context.

\section{WIDE FIELD MULTI APERTURE ARRAYS}

As seen from Table 1, Aperture Arrays for the SKA may operate up to about $1.4 \mathrm{GHz}$. The aperture array concept is a most flexible implementation of a telescope concept relies on full electronic pointing, dual polarized beam-forming techniques and algorithms that allow calibrated multi-beaming within the, possibly wide, single element array beam.

The potential for multi-object/multi-satellite reception can be inferred from the LOFAR observation shown in Figure 1. Using an astronomical application as example, it was recently demonstrated that 5 spatially widely separated pulsars can be observed simultaneously. In LOFAR, the single element beam pattern is essentially determined by a wide, all sky, dipole pattern with nulls at the horizon. As a result, all observed pulsars positioned far from the galactic plane are simultaneously observed in a single LOFAR observation at a frequency band around $70 \mathrm{MHz}$. Note that the processing requires the observations to be dedispersed to compensate for the pulse broadening through the intergalactic medium, a process essential for any coherent signal travelling through a dispersive medium.

While this experiment was performed at low frequencies less relevant to space applications it served to demonstrate the principle capability of aperture arrays. At these frequencies LOFAR performs as a socalled sparse array in which the element spacing is wider than half a wavelength [2].

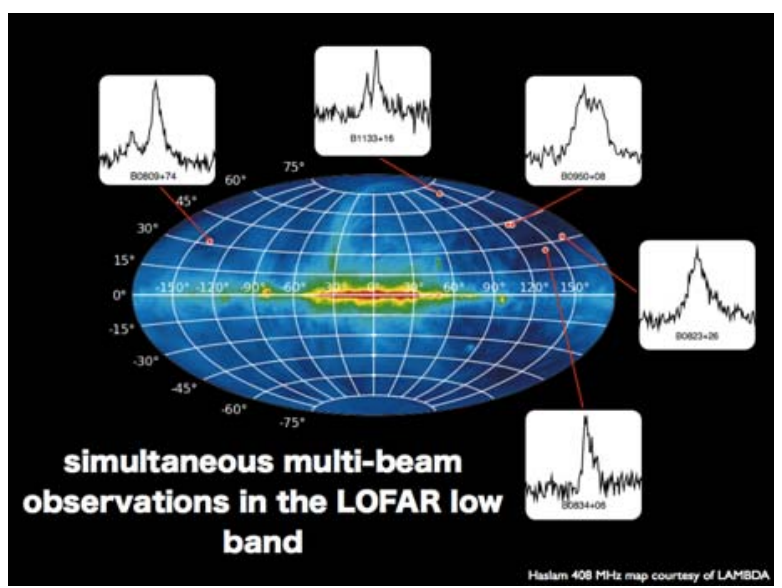

Figure 1: Demonstration with LOFAR of simultaneous multibeaming capability on five spatially widely separated pulsars. While the experiment was performed around $70 \mathrm{MHz}$ it served to demonstrate the principle capability of aperture arrays.

At higher frequencies, aperture arrays are best arranged as dense arrays with element spacing (much) less than half a wavelength. When designed properly, this also constitutes a constant collecting area with wide frequency band observing capability of $f_{\max } / f_{\min }>3$. Figure 2 shows a demonstrator arrangement as a step to 
prove the feasibility of the concept for radio astronomy and the SKA. Designated EMBRACE [11], it has two stations one in the Netherlands at the Westerbork Radio Observatory with a collecting area of about $150 \mathrm{~m}^{2}$ (as shown in the picture) and another at Nancay Radio Observatory in France. EMBRACE operates in the frequency band $500-1500 \mathrm{MHz}$ and the electronics allows for two fully independently steerable beams on the sky.

In this case the electronic processing is arranged such as to make an image of part of the galactic plane to be made in one beam while the other beam allowed to make a pulsar observation simultaneously and

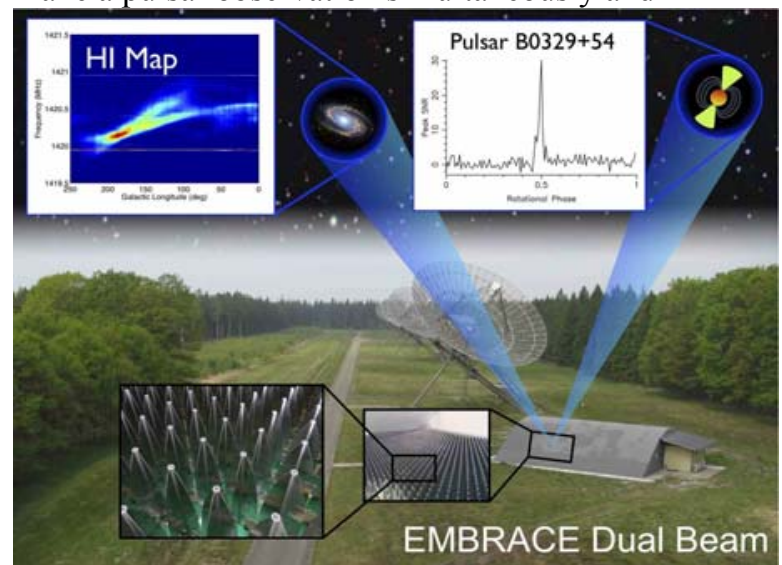

Figure 2: Demonstration with EMBRACE of simultaneous multi-beaming capability on two spatially widely separated independent beams at different frequencies around $1.4 \mathrm{GHz}$. The experiment served to demonstrate the principle capability of dense aperture arrays for radio astronomy, but it also shows prospects for space related applications outside the astronomy context.

independently. While intended for radio astronomy, the prospect for space related applications outside the astronomy context can be envisaged.

\section{WIDE FIELD FOCAL PLANE ARRAYS RESULTS}

The SKA aims to have reflectors at frequencies where aperture arrays are costwise unaffordable or yet unproven. At several places around the world but notably in the Netherlands [6] and Australia [9], the concept of dense arrays as focal plane feed systems are tested and implemented. Again, these allow for widely separated beams on the sky albeit within the reflectors Field of View which even for low (effective) Focal plane to Diameter ("F/D") ratios can be much larger than a single diffracted beam.

In Figure 3 the potential of enhancing the single telescope reflectors capability is demonstrated using a

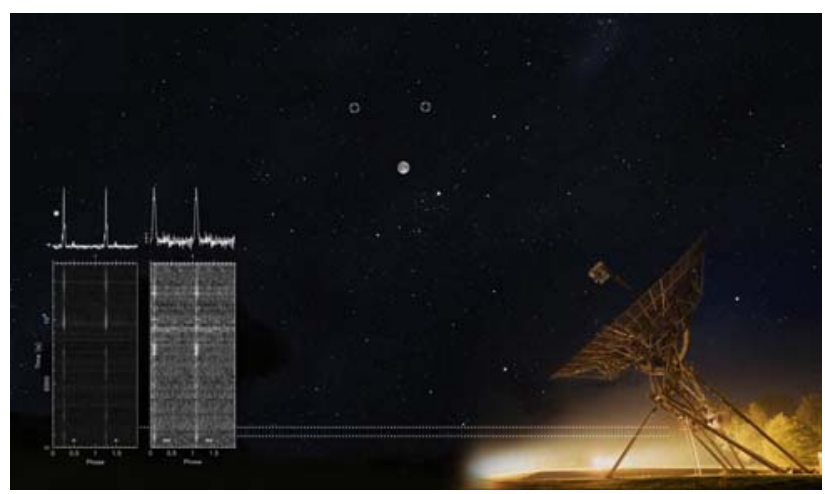

Figure 3: Demonstration with a single 25m Antenna of the Westerbork Synthesis Radio Telescope in the Netherlands, of two pulsars about 3.5 degrees or 7 moon diameters apart observed simultaneously around $1.4 \mathrm{GHz}$ with a focal plane array feed system. The observations demonstrated the huge potential for enhancing the observing capability of single reflector systems.

single $25 \mathrm{~m}$ reflector of the Westerbork Radio Synthesis Telescope equipped with a focal plane array (PAF) as part of the APERTIF program. Two pulsars about 7 diffracted beamwidth apart are observed simultaneously. In this case a single diffracted beamwidth at the observing frequency of about $1.4 \mathrm{GHz}$ corresponds to the moon angular diameter of 0.5 degrees. In APERTIF aims to equip $12 \mathrm{WSRT}$ telescopes with PAF systems. The beam-forming (37 beams) and correlation of the telescope beams will be based on a very flexible very high throughput digital system based on the UniBoard signal processing boards [21].

Systems like these are non-trivial to realize but their feasibility is proven and implementable to relatively low incremental cost on existing e.g. tracking infrastructure. The added capability is obviously multi-satellite and multi-object observing/tracking with large angular separation and high efficiency.

For example, the focal plane feed arrangement in Figure 3 , has a measured illumination efficiency increase of over $15 \%$ over a large frequency band with the reflectors approximate $\mathrm{F} / \mathrm{D}$ ratio of 0.43 .

As the SKA is planned to operate up to $10 \mathrm{GHz}$ with arrays of $>2000$ reflectors each of about $15 \mathrm{~m}$ diameter, wideband feeds and mass producible dishes are being explored [e.g. 8, 19].

The composite of the array of (so many) smaller dishes constitute a flexible and sensitive instrument with a relative large primary field of view i.e. determined by the dish diameter.

The (combination of the) characteristics mentioned above translate directly into increased potential for solar system, satellite and radio science missions. 


\section{NON-ASTRONOMICAL POTENTIAL}

Early on, it was realized that the SKA could be used for non-astronomy applications as well. For example, in [14, ch 47] there is a chapter on (deep space) spacecraft tracking with the SKA due to the planned high angular resolution and high sensitivity potential. Also in [16] the emphasis was on Space craft tracking applications. High precision tracking was demonstrated using existing radio telescopes through the use of Very Long Baseline Interferometry ("VLBI") techniques in the successful descent trajectory measurements of the Huygens probe in Titan's atmosphere [17].

Using radio-interferometry the angular position of the emitting radio source can be measured with the approximate accuracy in Eq.1.

$$
\Delta \phi=\frac{\lambda}{S N R * B l}
$$

where $\lambda$ is the signal wavelength, $\mathrm{Bl}$ is the interferometer baseline and SNR the signal-to-noise ratio of the detected interferometer response. At $\mathrm{S}$ band and a baseline of the earth diameter, the corresponding resolution is of order milli-arcsecond and with SNR $\sim 20$, this allows for sub-km accuracy at the distance of Titan's probe [17]. With the SKA the resolution will not increase but its sensitivity will, assuming compound beams ("tied array") can be made.

In [18] the value of a large ground array, and the SKA in particular, for telemetry reception from distant and low power probes is discussed in detail, in this case also vis a vis the developments in NASA's Deep Space Network.

As with [16] with regard to Titans atmosphere, precise tracking based on high resolution Doppler analysis opens up perspectives for study of planetary and solar system science in general. Besides the value of an SKAscale array for planetary radio observations, radio science measurements using the Earth-spacecraft radio link and precision Doppler measurements can be used to determine the gravitational field of a solar system object as well.

\section{POTENTIAL FOR PLANETARY OBSERVATIONS AND RADIO SCIENCE}

Larger sensitivity translate directly into increased range for planetary radar observations.

For example, follow-up observations will be possible for a larger class of Near-Earth asteroids, including precise orbit determination, radar imaging and shape reconstruction important for impact hazard and pure science.

With the full SKA sensitivity and size, radar studies could be undertaken of the moons of Uranus and
Neptune/Triton and larger Kuiper belt objects.

High angular resolution radar surveys could also include improved understanding of Earth orbital debris for current and future spacecraft. These may not require full and continuous observations and possibly allow for reduced sensitivity and reduced resolution follow-ups.

The SKA could be used in support of bi-static radar at $\mathrm{S}$ - and X-band respectively as both are within the SKA frequency range. Alternatively, migration to a $4 \mathrm{x}$ higher frequency i.e. at the $34 \mathrm{GHz}$ Deep Space uplink band, not only requires smaller dishes for the same radar sensitivity performance, but also moves into the range of arrays of smaller dishes of $<35 \mathrm{~m}$ class.

For radioscience applications, the sensitivity of the SKA will allow using signals to be measured from low power and/or simple multiple spacecrafts or landers. The ability to track multi-planet orbiters and/or landers, is relevant to improve orbital dynamics and improve on models of the gravitational potential. Also multi-beam capability explored by the SKA as mentioned in section 3 may support spacecraft tracking relevant for future satellite cluster missions.

As in present day aperture synthesis observations, the SKA will be designed for accurate amplitude and phase calibration and highly stable frequency stability in general required to achieve high resolution and imaging astronomy using coherent receptors [e.g. 15]. However, the SKA will also be designed specifically for high dynamic range imaging of 10 million to 1 often defined as the maximum power ratio within the imaged map. This requires extremely stable instrument performance and known stable beamshapes based on optimal station configuration e.g. for ionospheric calibration techniques and clever algorithms all elements of study now.

It is likely that the results are relevant for radio science applications in space as well. In support for increased observing performance in present and future radio astronomy interferometry, the mathematical foundation is laid down in the so called measurement equation using Jones matrices [e.g. 20].

\section{CONCLUSIONS}

The SKA, its technologies and its concept approaches offer potential for future planetary, and multi spacecraft missions as well for the study of radio science aspects. Largely, these capabilities are induced because of resolution, sensitivity, multi aperture- and multi-beam arrays and signal processing techniques.

En route to the final SKA, the present capabilities of widefield techniques have been presented based on demonstrator results in support of the envisaged applications. 


\section{ACKNOWLEDGEMENTS}

The authors wish to acknowledge the use of material from Tom Hassall and the LOFAR Pulsar Group, the EMBRACE team and Joeri van Leeuwen, Jason Hessels, Wim van Cappelllen and the APERTIF team and the contribution of material supplied by Dr. D. L. Jones at JPL.

\section{REFERENCES}

1. Dewdney, P.E., Hall, P.J., Schilizzi, R.T. \& Lazio, T.J.L.W. (2009).The Square Kilometre Array, Proc. IEEE, Vol. 97(8), pp1482-1496

2. van Ardenne, A., Bregman, J.D., van Cappellen, W.A., Kant, G.W. \& bij de Vaate, J.G. (2009). Extending the Field of View with Phased Array Techniques. Proc. IEEE, vol.97(8), pp1531-1542

3. "Square Kilometre Array Design Studies, SKADS", www.skads-eu.org

4. Torchinsky, $\mathrm{S}$ et al. (2010). Proc. SKADS Conference, Limelette Oct. 2009, pp 9-14, ISBN 978-90-805434-5-4

5. Faulkner, A.J. et al.(2010). Aperture Arrays for the SKA - the SKADS White Paper, SKA Memo 122: www.skatelescope.org

6. Ivashina,M.V. et al. (2011). An optimal Beamforming Strategy for Wide-filed Surveys with Phased-Array-Fed Reflector Antennas. IEEE-AP vol. 59(6), pp1864-1875

7. Yang, J. et al. (2011). Cryogenic 2-13GHz Eleven feed for Reflector Antennas in future Wideband Radio Telescopes. IEEE-AP vol. 59(6), pp19181934

8. Jonas, J.L.(2009). MeerKAT-The South African Array with Composite Dishes and Wide-band Single Pixel Feeds. Proc. IEEE, Vol. 97(8), pp1522-1530

9. De Boer, D.B. et al.(2009). Australian SKA Pathfinder: A high dynamic range Wide Field of View Survey Telescope. Proc. IEEE, Vol. 97(8), pp1507-1521

10. de Vos, M., Gunst, A.W. \& Nijboer, R.(2009).The LOFAR Telescope: System Architecture and Signal Processing. Proc. IEEE, Vol. 97(8), pp1431-1437

11. Kant, G.W. et al.(2011). EMBRACE: A Multibeam 20.000 element Radio Astronomical Phased Array Antenna Demonstrator. IEEE-AP vol. 59(6), pp1990-2003

12. C. J. Lonsdale, C.J. et.al.(2009). The Murchison
Widefield Array: Design Overview. Proc. IEEE, Vol. 97(8), pp1497-1506

13. “Aperture Array Verification Program”, www.skaaavp.eu

14. Carilli, C. \& Rawlings, S.(eds.) (2004). Science with the Square Kilometre Array. New Astron. Rev., Vol 48

15. Leshem, A. et al. (eds.) (2008). IEEE Journal of selected topics in Signal Processing, Vol2(5)

16. Bij de Vaate, J.G., Gurvits, L.I., Progrebenko, S.V. \& van 't Klooster, C.G.M. (2004). Spacecraft Tracking Applications of the Square Kilometre Array. Proc. ESA 3td Int. Worshop on TTC systems for Space Applications, pp 93-100

17. Pogrebenko, S. V., Gurvits, L. I., Campbell, R. M., Avruch, I. M., Lebreton, J.-P., \& van't Klooster, C. G. M. (2004). VLBI tracking of the Huygens probe in the atmosphere of Titan. Proc. Int. Workshop Planetary Probe Atmospheric Entry and Descent Trajectory Analysis and Science, ESA SP-544, ISBN 92-9092-855-7, 2004, p. 197 - 204

18. Deutsch,L.J., Preston, R.A. \& Vrotsos, R. (2010). The future of NASA's DSN and Applications to Planetary Probe Missions. Proc. Int. Planetary Probe Workshop

19. Ivashina, M.V. et al. (2011). An Axi-symmetric Segmented Composite SKA Dish Design: Performance and Production Analysis. Proc. AsiaPacific Micr. Conf. (to be published), Dec., Austr.

20. Smirnov, O.M. (2011). Revisiting the Radio Interferometer Measurement Equation. Series of articles in Astronomy \& Astrophysics, Vol 527

21.Szomoru, A. (2010. The UniBoard. $10^{\text {th }}$ EuropeanVLBI Network Symp. And EVN Users Meeting: VLBI and the new generation of radio arrays, Man. 20-24, PoS (10 EVN Symp.) 098 